\title{
Non-contrast CT Image Automatic Diagnosis of Large Hemispheric Infarction in Hyper-acute Phase Based on Convolutional Neural Network
}

\author{
Chengjian Huang \\ Shenzhen Institute of Advanced Technology, Chinese \\ Academy of Sciences, Shenzhen, China and University of \\ Chinese Academy of Sciences, Beijing, China \\ cj.huang@siat.ac.cn \\ Haizhou Sun \\ The College of IoT Engineering, Hohai University, \\ Changzhou, China \\ 874302225@qq.com
}

\author{
Min Liu \\ Linyi People's Hospital, Department of Radiology, Linyi, \\ China \\ 1223834936@qq.com
}

\section{Qingmao $\mathrm{Hu}^{*}$}

Shenzhen Institute of Advanced Technology, Chinese Academy of Sciences, Shenzhen 518055, China and CAS Key Laboratory of Human-Machine Intelligence-Synergy Systems, Shenzhen Institute of Advanced Technology, Chinese Academy of Sciences, Shenzhen, ChinaSchool of Artificial Intelligence, University of Chinese Academy of Sciences, Beijing, China qm.hu@siat.ac.cn

\begin{abstract}
Hyper-acute large hemispheric infarction (LHI) is a serious type of cerebral infarction, characterized by sudden onset and rapid worsening. Without prompt diagnosis and treatment, it can severely endanger patient health and life. Therefore, early diagnosis of hyperacute cerebral infarction and active treatment are very important. Clinically, non-contrast computed tomography (NCCT) is the preferred modality to quickly diagnose and evaluate suspected stroke patients. In this work, we proposed and evaluated an automatic deep learning method for segmenting ischemia and recognizing LHI in the hyper-acute phase from NCCT images. The method was validated against 135 NCCT volumes to attain an accuracy of $91.67 \%$, precision of $90.95 \%$, recall of $91.67 \%$, and area under the receiver operating curve of 0.89 in detecting LHI at patient level. The proposed method could be potentially employed clinically to identify LHI in the hyper-acute phase quickly and accurately to aid decision making on effective clinical treatment for ischemic stroke.
\end{abstract}

\section{CCS CONCEPTS}

- Computing methodologies; • Artificial intelligence; • Computer vision; • Computer vision problems; • Interest point and salient region detections;

${ }^{*}$ Corresponding author. Tel.: +86-755-86392214; fax: +86-755-86392299, E-mail address: qm.hu@siat.ac.cn

Permission to make digital or hard copies of all or part of this work for personal or classroom use is granted without fee provided that copies are not made or distributed for profit or commercial advantage and that copies bear this notice and the full citation on the first page. Copyrights for components of this work owned by others than ACM must be honored. Abstracting with credit is permitted. To copy otherwise, or republish, to post on servers or to redistribute to lists, requires prior specific permission and/or a fee. Request permissions from permissions@acm.org.

AIPR 2021, September 24-26, 2021, Xiamen, China

(C) 2021 Association for Computing Machinery.

ACM ISBN 978-1-4503-8408-7/21/09..\$15.00

https://doi.org/10.1145/3488933.3488945

\section{KEYWORDS}

Large hemispheric infarction, Non-contrast CT, Hyper-acute stroke, Infarct segmentation, Convolutional neural network

ACM Reference Format:

Chengjian Huang, Min Liu, Haizhou Sun, and Qingmao Hu. 2021. Noncontrast CT Image Automatic Diagnosis of Large Hemispheric Infarction in Hyper-acute Phase Based on Convolutional Neural Network. In 2021 th International Conference on Artificial Intelligence and Pattern Recognition (AIPR 2021), September 24-26, 2021, Xiamen, China. ACM, New York, NY, USA, 8 pages. https://doi.org/10.1145/3488933.3488945

\section{INTRODUCTION}

Ischemic stroke is a pathological event caused by the interruption of blood supply to brain tissue, which is an important cause of death in adults [1]. Once an ischemic stroke occurs, human nerve tissue will die quickly over time, and rapid intervention is required. Large hemispheric infarction (LHI) is an important type of stroke, accounting for about $10 \%$ of all cerebral infarctions [2]. As many as $1 / 3$ of patients have neurological deterioration within 24 hours after the onset of disease. The case fatality rate of conservative medical therapy is about $41-80 \%$, and the prognosis is extremely poor [2, 3]. The current treatment guidelines of ischemic stroke are based on onset time. In chronological order, brain damage caused by ischemic stroke goes through four stages: hyper-acute phase (0-6 hours), acute phase (6-24 hours), sub-acute phase ( 24 hours- 2 weeks) and chronic phase (more than 2 weeks). Some randomized controlled studies have shown $[4,5]$ that decompressive craniectomy within 48 hours can reduce the mortality of patients. Therefore, identifying LHI during the hyper-acute period is beneficial to the risk stratification of patients and provide precise guidance for developing treatment plans and prognostic evaluation.

Radiomics plays an important role in the early prediction of the phase of LHI, and it is an important reference for early diagnosis, differential diagnosis and treatment. With the advancement 
of imaging technology and the popularization of imaging examinations such as head magnetic resonance imaging (MRI), we can obtain more information about the lesion. Neuroimaging examination within 6 hours of onset showed that infarct volume two-thirds larger than the middle cerebral artery blood supply area, early space-occupying effect, involved ipsilateral anterior cerebral artery or (and) posterior cerebral artery blood supply area, and internal carotid artery occlusion may be predictors of LHI. Because MRI and computed tomography (CT) perfusion (CTP) imaging have high sensitivity for detecting cerebral infarction, most ischemic brain image analyses are based on MRI or CTP. According to clinical data [6], MRI or CTP infarct area larger than $82 \mathrm{~cm}^{3}$ can be medically judged as LHI. Chen et al [7], used a set of 2 convolutional neural networks (CNNs) to segment diffusion weighted image (DWI) lesions with a lesion detection rate of $94 \%$. Guerrero et al. [8] used CTP images to develop a 3D CNN to estimate the size and location of acute infarct lesions, with an average Dice score of $49 \%$.

Although the data based on MRI and CTP are of high sensitivity, their relatively long imaging time limits their utility for rapid diagnosis and evaluation in the hyper-acute setting. On the contrary, non-contrast CT (NCCT) is fast, low-cost, more accessible, and is the first modality in the hyper-acute setting. However, because hyper-acute ischemia shows subtle changes in low-attenuation signals in NCCT images, NCCT has poor sensitivity to hyper-acute cerebral infarction which is yet to overcome. Tang et al. [9] employed a circular adaptive region of interest to extract multiple image features to detect early ischemic infarction of small lesions, without experimental validation on LHI. Boers et al. [10] used an intensity-based semi-automatic area growth algorithm (manually placed seed points and midline) to segment the infarct volume in subsequent NCCT images of 34 consecutive patients with acute ischemic stroke.

In short, currently there are few studies on analysing hyper-acute ischemia from NCCT images mainly due to the poor sensitivity. But Qiu et al. [11] provides a research basis to automatically detect LHI. In their study, the infarct volume segmented from MRI image has a good correlation with the infarct volume segmented from NCCT images, with the average difference between the two infarct volumes being $11 \mathrm{~cm}^{3}$, which indicates a good correlation. Accurate segmentation of the ischemia is conducive to the accurate diagnosis of LHI patients, so the work of this study focuses on improving the accuracy of the segmentation of the cerebral ischemia from NCCT images.

In this paper, we propose a computer-aided detection (CAD) scheme to quickly and accurately detect LHI from hyperacute NCCT images. We used the trained CNN segmentation model to automatically segment the infarct region and calculate the volume of the infarct core. By using an ischemic core $71 \mathrm{~cm}^{3}[7,11]$ as the threshold point, LHI patients could be distinguished. We evaluated the accuracy of the proposed CNN segmentation network to obtain a Dice coefficient of $90.36 \%$. We also used the hyper-acute LHI NCCT images to evaluate the proposed CAD scheme, and obtained an accuracy of $91.67 \%$.

\section{MATERIALS AND METHODS}

The proposed CAD scheme is carried out through three main steps (Figure 1). Firstly, we used the segmentation dataset to train the proposed segmentation $\mathrm{CNN}$ to obtain the probability map of the infarct area. Secondly, we used the trained segmentation CNN network to process LHI dataset to obtain a segmentation map of the infarct region. Finally, the volume of the lesion is obtained by calculating the segmentation map, and then the classification is performed with $71 \mathrm{~cm}^{3}$ as the threshold value to obtain the discrimination result of LHI.

\subsection{Datasets}

We collected two datasets: the segmentation dataset and the LHI dataset in the hyper-acute phase. The segmentation dataset is used to train and verify the CNN segmentation network, and the hyper-acute phase LHI dataset is used to verify the reliability of the research method in this paper. In the segmentation dataset, 45 hyper-acute ischemic patients (within 6 hours of stroke onset) were included. The infarct area was marked on the axial slices by experienced clinicians using the MITK Workbench software (https://www.mitk.org). This is used as a reference for segmentation of the infarct region, and verified by a professional neuroradiologist. The LHI dataset included 135 NCCT imaging data, including 10 healthy volunteers, $40 \mathrm{LHI}$ patients and 85 non-LHI cerebral infarction patients. The size of these NCCT images is $512 \times 512 \times(24 \sim 32)$ and the voxel spacing is $0.43 \times 0.43 \times 4.1 \mathrm{~mm}^{3}$ to $0.43 \times 0.43 \times 4.8 \mathrm{~mm}^{3}$.

These datasets collected from Huizhou People's Hospital and Linyi People's Hospital. Both hospitals have been involved in in a National Stroke Registry since 2005, which registered patients prospectively with stroke ictus within 6 hours according to a preestablished system. The protocol of the research has been approved by the Institutional Review Board of both hospitals. All patients gave written consent and provided permission for scientific and educational purpose.

\subsection{Data Preparation and Expansion}

In NCCT images of the head, the Hounsfield Unit (HU) of each tissue is quite different. It is necessary to select a window to better represent the ischemic area in the brain parenchyma. In this experiment, we selected a window value of $-30-100 \mathrm{HU}$, so that the network can better extract image features. When only a few training samples are available, the increase of data is crucial for the generalization and robustness of neural networks [12]. Unfortunately, there are too few ischemia data available for training in our experiments. Therefore, we considered using data enhancement. Because there has been experience showing that image transformation is effective in image recognition tasks [13], we performed geometric transformations such as random cropping, rotation, and displacement on each image slice. We also randomly perform operations such as blurring, sharpening, distortion and noise addition on the axial slices with a $50 \%$ probability. To cope with the extremely unbalance of ischemia and healthy tissues we augmented axial slices differently: augmenting axial slices with ischemia 5 times and those without ischemia only 1 time.

\subsection{Segment Networks}

We adopted the form of "encoding-decoding" in the overall network structure (Figure 2). Typical structure of the convolutional network was used in the encoder, and a non-local attention module [13] was 


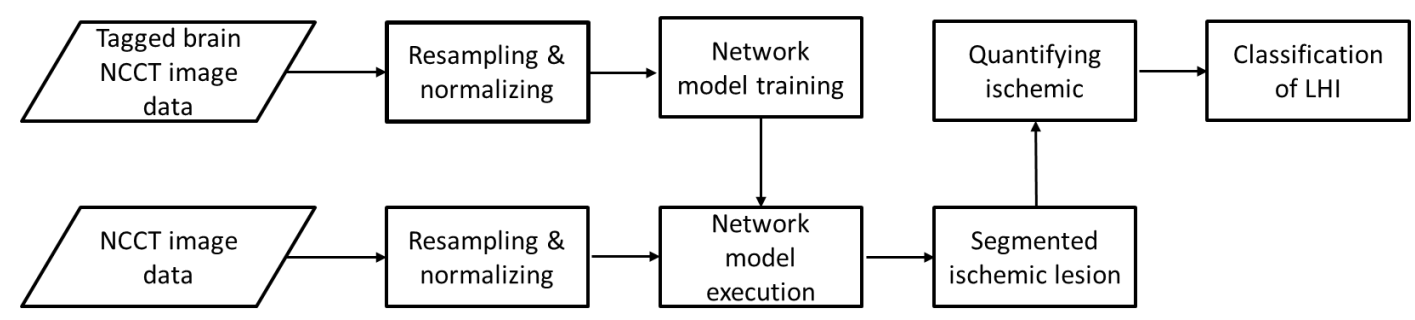

Figure 1: Flow chart of the experimental method.

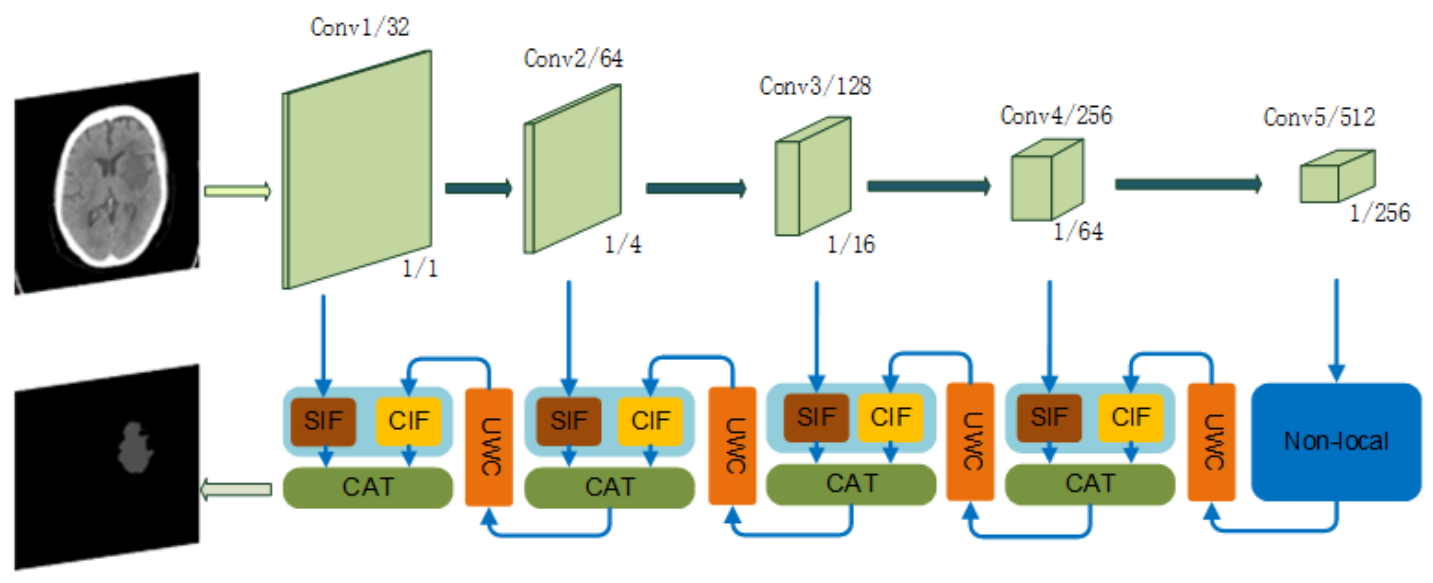

Figure 2: Overview of our proposed model. SIF: spatial information fusion unit, CIF: channel information fusion unit, UWC: upsampling with convolution unit, CAT: concatenate unit, Non-local: non-local attention unit, Conv $i / c$ represents the characteristics of coutput channels extracted by the encoder in the $i-t h$ stage, $1 / N$ for down-sampled to $1 / N$ of the original scale.

added to enhance the global context information. Two information fusion modules are used in the decoder to fuse the spatial information in the encoder and the channel information in the decoder to enhance the ability to express feature information.

2.3.1 Encoder. The encoder is designed to extract image features through four "convolution-convolution-pooling" operations, each of which involves repeated applications of two convolutions. After each convolution layer, there is a batch normalization layer [14] and a nonlinear activation function PReLu [12], with a maximum pooling operation after two convolutions. And after each pooling operation, we doubled the number of feature channels to extract richer image features.

The contralateral difference in brain NCCT images is often important discriminant information [15]. Since convolution and pooling operations both focus on local areas in the encoding process, global information is added through the non-local attention unit (Non-local) after the fourth "convolution-convolution-pooling" to increase the extraction of features [16]. When the unit calculates the response of a certain position, it will consider the weighting of the characteristics of all channel positions and spatial positions. In this way, the detection of cerebral ischemia areas can be improved and false positives can be suppressed.
2.3.2 Decoder. As the CT images are down-sampled, the spatial detail information of the deep feature output map is often lost, which reduces the accuracy of segmentation. To solve this problem, this paper designs a spatial information fusion module to generate spatial information from features of various scales obtained in the encoding structure, and inputs the spatial information to the decoder.

As shown in Figure 2, the decoding process consists of 4 steps. Firstly, the feature information extracted by the encoder is expanded by the upsampling with convolution (UWC) unit. Secondly, shallow features of the same resolution extracted by the decoding layer are used to extract the spatial feature information through the spatial information fusion (SIF) unit. Thirdly, deconvolution is used to obtain characteristic information of the channel through the channel information fusion (CIF) unit. Finally, the spatial feature information and channel feature information obtained above are concatenated, and convoluted in the concatenation unit (CAT). The result of CAT is inputted into next UWC unit and the above operations are repeated. It is noteworthy that, we halve the number of channels to correspond to the decoding structure after each deconvolution operation. After the feature passes through four deconvolution operations, the convolution is used to compress the feature information of all channels into a feature map with a channel dimension of 1 . Finally, the feature map is regressed 


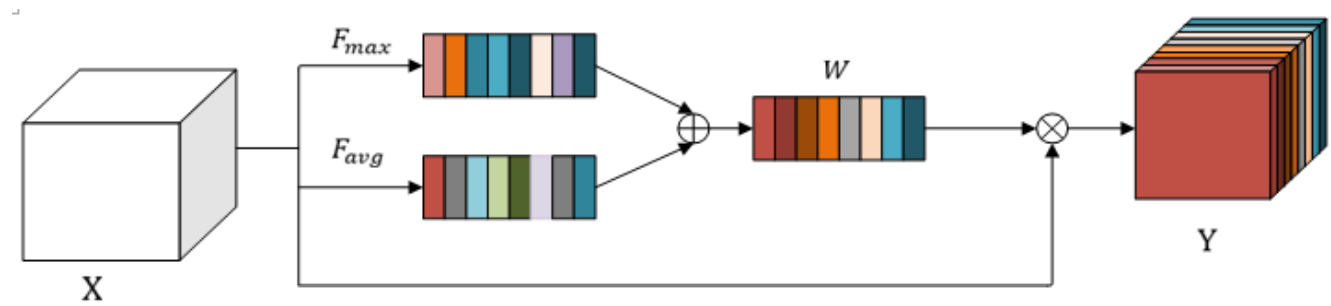

Figure 3: Channel information fusion unit.

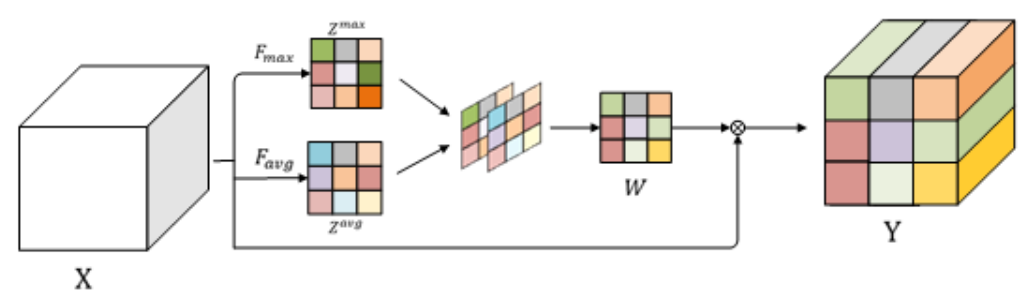

Figure 4: Spatial information fusion unit.

through the softmax to obtain an infarct probability map with the same resolution as the input image.

Channel Information Fusion Unit (CIF):Inspired by [17], this paper used global information to explicitly model the dynamic nonlinear dependencies between channels, simplify the learning process, and enhance the network's representation ability (Figure 3). To obtain its global information, we merge the information in each channel. In the Figure 3, the feature map $X \in \mathbb{R}^{H * W * C}$ is used as the input of the module. $S_{c} \in \mathbb{R}^{H * W}$ is the spatial information on thec-thchannel, and the spatial information $S_{c}$ of each channel is fused by the global maximum pooling and global average pooling to obtain the channel weight coefficient $\boldsymbol{h} \in \mathbb{R}^{C}$ and to calculate the weight $h_{c}$ of the $c$-thchannel:

$$
\begin{aligned}
h_{c}= & F_{a v g}\left(S_{c}\right)+F_{\max }\left(S_{c}\right) \\
& =\frac{1}{H \times W} \sum_{i=1}^{H} \sum_{j=1}^{W} S_{c}(i, j)+\max \left(\sum_{i=1}^{H} \sum_{j=1}^{W} S_{c}(i, j)\right)
\end{aligned}
$$

After obtaining the channel information weight, we used PReLU to activate it in order to control the excitation of each channel. But at the same time, the information between channels does not exist in isolation, and there is a non-linear interaction between the information. We consider using the "sigmoid" function to capture the correlation of the channel information after obtaining the channel information weight $\boldsymbol{h}$.By establishing the dependency relationship between channels, better adaptive recalibration of channel-related features and responses can be achieved. The calculation process is as follows:

$$
W=F_{r s}(\boldsymbol{h})=g(\sigma(\boldsymbol{h}))
$$

$\sigma$ and $g$ respectively represent PReLU transform and sigmoid transform. Finally, the obtained channel information weight $W \in \mathbb{R}^{C}$ is weighted onto the input feature map $\mathrm{X}$ to obtain the output $Y \in \mathbb{R}^{H * W * C}$ of the module. The calculation process is as follows:

$$
Y=X \otimes W
$$

Where $\otimes$ is multiplication pixel by pixel.

Spatial Information Fusion Unit (SIF):The low-resolution features obtained by the encoder after multiple convolutions and downsampling are often abstract features such as points, lines, and edge contours. These abstract features are conducive to the classification of objects [18]. However, in the segmentation of the infarct region, more accurate segmentation and positioning are needed, and the spatial information of the feature map is more useful. As the features of the shallower encoding layers contain richer spatial information, we extract the spatial information of the encoding layer and apply it to the decoder to improve the accuracy of segmentation (Figure 4).

In the convolution process, we use multiple convolution kernels to perform convolution operations on the input features to generate multi-channel feature maps. The spatial information contained in these feature maps is correlated and reflects the same location information. Therefore, we merge the position information of multiple channels into the same feature map. In the Figure 4, the feature $\operatorname{map} X \in \mathbb{R}^{H * W * C}$ is used as the input of the module. $L_{c} \in \mathbb{R}^{C}$ is the spatial information set with the spatial position of $S(i, j)$.The average and maximum values of the spatial information sets of each pixel of the feature map are calculated to obtain a feature map $Z^{a v g}$ and $Z^{\max }$, whose size is $H * W$.

$$
\begin{aligned}
& Z_{(i, j)}^{a v g}=F_{a v g}\left(L_{c}\right)=\frac{1}{C} \sum_{c=1}^{C} l_{c} \\
& Z_{(i, j)}^{\max }=F_{\max }\left(L_{c}\right)=\max \left(L_{C}\right)
\end{aligned}
$$

Then we simply stack the two obtained feature maps $Z^{a v g}$ and $Z^{a v g}$, activate the PReLu function through a1 $\times 1$ convolution to obtain a feature map of size $H * W$, and use the "sigmoid" transformation to map it to $[0,1]$ get the final spatial information weight $W \in \mathbb{R}^{H * W}$. Finally, the obtained spatial information weight $W \in \mathbb{R}^{C}$ is weighted 
onto the input feature mapX to obtain the output $Y \in \mathbb{R}^{H * W * C}$ of the module. Formula (3) is used to get $Y$ from $X$ and $W$.

Upsampling with Convolution Unit (UWC): The feature information of the upper layer is deconvolved to obtain a feature map with higher resolution. At the same time, two convolutional layers are used to halve the number of feature information channels to reduce information redundancy.

Concatenate Unit, (CAT): The CAT unit simply connects the channel information obtained from CIF unit and the spatial information obtained from SIF unit.

2.3.3 Loss Function. In medical image segmentation tasks, Dice loss is the most widely used loss function, which is used to measure the difference between the prediction result and the gold standard. With this method, the evaluation criteria can be directly optimized and can achieve higher accuracy. However, with regard to the segmentation of cerebral ischemic regions, the ischemic region often occupies a smaller part of the entire image, which results in an extremely unbalanced data category. We hope to weaken this imbalance and improve the recall rate of pixel classification at the expense of certain accuracy. Therefore, we introduced the Tversky loss function [19] as the loss function for network training. The calculation formula is as follows:

$L_{T}=1-\frac{\sum_{i-1}^{N} p\left(x_{i c}\right) y_{i c}+\varepsilon}{\sum_{i-1}^{N} p\left(x_{i c}\right) y_{i c}+\alpha \sum_{i-1}^{N} p\left(x_{i c}\right) y_{i \bar{c}}+\beta \sum_{i-1}^{N} p\left(x_{i \bar{c}}\right) y_{i c}+\varepsilon}$

In the formula (6), $x_{i c}$ represents the pixel $i$ of the input image, $p\left(x_{i c}\right) \in[0,1]$ represents the probability that the predicted result is the foreground areac, $p\left(x_{i \bar{c}}\right) \in[0,1]$ represents the probability that the predicted result of the pixel $i$ is the non-foreground area $\bar{c}, y_{i c} \in[0,1]$ represents the ground truth of $c$, and $\varepsilon$ is a hyperparameter that prevents the denominator from being equal to zero. Among them, $\alpha$ and $\beta$ are the balance factors. From the formula, it can be found that when $\alpha=\beta=0.5$, the Tversky loss becomes the Dice coefficient. Therefore, we adjust the balance factor to flexibly balance false negatives and false positives. In the experiment, we found that $\alpha=0.3$ and $\beta=0.7$ could yield best results.

\subsection{Classification}

Firstly, we consider pixels with a probability more than 0.5 as is chemic pixels, and the other as background pixels, thus we get a segmentation map of the ischemic area. Secondly, we use the segmentation map of all pixels to calculate the 3D connected components in the segmentation map and remove small connected components to reduce the influence of false positives. Thirdly, we count the sum of the pixels of all the remaining connected components and obtain the ischemic volume based on the actual voxel value. Finally, we consider patients with an ischemic volume more than $71 \mathrm{~cm}^{3}$ as LHI patients according to [11].

\section{EXPERIMENTS AND RESULTS}

\subsection{Experimental Details and Parameter Setting}

The algorithm was implemented based on the Keras framework and Tensorflow backend Python language, and four TITAN RTX GPUs with $24 \mathrm{G}$ video memory are used for training. $85 \%$ of the training data is randomly assigned as training set, with the remaining being validation set. The network was trained for 50 epochs using the training set data, and the batch_size was set to 16 . The adaptive moment estimation (Adam) optimizer [20] was used to train the segmentation network, where parameters were set as follows: beta_1 $1=0.9$, beta_2 $=0.999$, epsilon $=10 \mathrm{E}-8$, and the initial learning rate $=10 \mathrm{E}-4$. In each round, a 0.9 th power polynomial decay is performed. By changing a specific parameter while leaving other parameters unchanged, the validation set was used to adjust all parameters for evaluation. During the test phase, the parameters of all networks are kept constant.

\subsection{Segmentation Dataset of Hyper-acute Ischemic Stroke}

We first conduct experiments on the segmentation dataset to verify the proposed network structure. We compare with other benchmark network structures for image segmentation, such as: U-net [11], Segnet [21], deeplabv3+ [22], U-net++ [23]. Different evaluation indicators were used to verify the effectiveness of the evaluation results. In order to ensure fairness, we conducted comparative experiments under the same experimental preparation and experimental details. At the same time, we also adjusted the parameters of these comparison methods to the best. The segmentation effect comparison was shown in Figure 5, and the evaluation results were shown in Table 1 and Table 2. Four metrics were used to evaluate the detection and segmentation results: precision rate, recall rate, Dice similarity coefficient (DSC), and IOU coefficient (Intersection over union). We use accuracy and recall to evaluate whether the model accurately detects cerebral ischemia in the slice (Table 1). The DSC and IOU coefficients are used to evaluate the accuracy of segmentation (Table 2). The calculation formula are as follows:

$$
\begin{gathered}
\text { Precision }=\frac{T P}{T P+F P} \\
\text { Recall }=\frac{T P}{T P+F N} \\
D S C=\frac{2 \times T P}{2 \times T P+F P+F N} \\
I O U=\frac{T P}{T P+F P+F N}
\end{gathered}
$$

Where TP, FP, and FN are the numbers of true positive (foreground being predicted as foreground), false positive (background being predicted as foreground), and false negative (foreground being predicted as background).

Segmentation of the relevant methods on cerebral ischemia slices was shown in Figure 5. We also compare each segmentation network with evaluation metrics in Table 1 and Table 2. At the same time, in order to illustrate the effectiveness of the modules used in the network, we conduct ablation experiments on the basic network. The comparison results were summarized in Table 3 and Table 4

\subsection{Hyper-acute HLI dataset}

We verify the CAD scheme on the HLI dataset. The comparative results are summarized in Table 5 


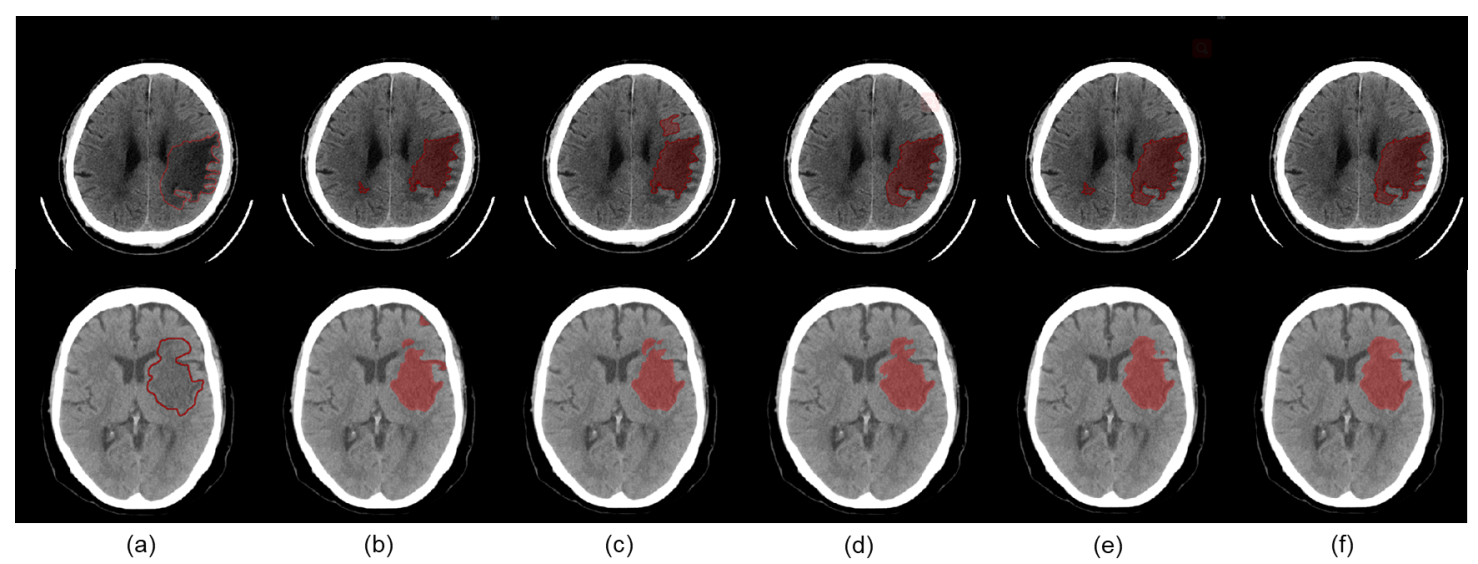

Figure 5: The ischemic region segmentation results of the hyper-acute cerebral ischemia dataset under different methods. (a) Manual standard; (b) U-net; (c) Segnet; (d) deeplabv3+; (e) Unet++; (f) Ours.

Table 1: Detection results of different methods on the segmentation dataset

\begin{tabular}{lll}
\hline Method & Precision $(\%)$ & Recall $(\%)$ \\
\hline U-net [12] & 71.42 & 90.70 \\
Segnet [21] & 76.27 & 88.35 \\
Deeplabv3+ [22] & $\mathbf{9 2 . 4 7}$ & 86.00 \\
Unet++ [23] & 88.89 & 96.00 \\
Ours & 88.25 & $\mathbf{9 8 . 0 7}$ \\
\hline
\end{tabular}

Table 2: Segmentation results of different methods on the segmentation dataset

\begin{tabular}{lll}
\hline Method & DSC $(\%)$ & IOU $(\%)$ \\
\hline U-net [12] & $84.64 \pm 29.70$ & $81.19 \pm 30.77$ \\
Segnet [21] & $83.38 \pm 31.60$ & $80.12 \pm 32.12$ \\
Deeplabv3+ [22] & $87.12 \pm 26.81$ & $83,72 \pm 27.99$ \\
Unet++ [23] & $89.46 \pm 21.90$ & $85.68 \pm 24.43$ \\
Ours & $\mathbf{9 0 . 3 6} \pm \mathbf{2 1 . 3 1}$ & $\mathbf{8 6 . 7 5} \pm \mathbf{2 2 . 8 2}$ \\
\hline
\end{tabular}

Table 3: Ablation study of the proposed modules on the detection dataset

\begin{tabular}{lll}
\hline Method & Precision $(\%)$ & Recall $(\%)$ \\
\hline Base(U-net) & 71.42 & 90.70 \\
Base + Non-local & 76.47 & 95.59 \\
Base + Non-local +CIF & 80.23 & 97.82 \\
Base+Non-local+CIF+SIF & $\mathbf{8 8 . 2 5}$ & $\mathbf{9 8 . 0 7}$ \\
\hline
\end{tabular}

Table 4: Ablation study of the proposed modules on the segmentation dataset

\begin{tabular}{lll}
\hline Method & DSC (\%) & IOU (\%) \\
\hline Base(U-net) & $84.64 \pm 29.70$ & $81.19 \pm 30.77$ \\
Base + Non-local & $87.38 \pm 25.02$ & $83.02 \pm 23.74$ \\
Base + Non-local +CIF & $88.62 \pm 22.97$ & $84.32 \pm 24.43$ \\
Base+Non-local+CIF+SIF & $\mathbf{9 0 . 3 6} \pm \mathbf{2 1 . 3 1}$ & $\mathbf{8 6 . 7 5} \pm \mathbf{2 2 . 8 2}$ \\
\hline
\end{tabular}


Table 5: Classification of different methods on the hyper-acute HLI dataset

\begin{tabular}{lllll}
\hline Method & Precision (\%) & Recall (\%) & Accuracy (\%) & AUC \\
\hline U-net [12] & 87.89 & 89.39 & 89.09 & 0.87 \\
Segnet [21] & 88.38 & 88.63 & 88.63 & 0.86 \\
Deeplabv3+ [22] & 89.39 & 89.18 & 89.30 & 0.88 \\
Unet++ [23] & 89.96 & 90.90 & 90.15 & 0.88 \\
Ours & $\mathbf{9 0 . 9 5}$ & $\mathbf{9 1 . 6 7}$ & $\mathbf{9 1 . 6 7}$ & $\mathbf{0 . 8 9}$ \\
\hline
\end{tabular}

\section{DISCUSSION AND CONCLUSION}

From Table 1 and Table 2, it can be seen that deep learning can perform well in the detection and segmentation of hyper-acute cerebral ischemia. Segnet, which is also based on the "encodingdecoding" structure, has inferior detection and segmentation performance to ours. The reason is that the information used by Segnet is the pooled information of the encoder without appropriate spatial information. Compared to Segnet, U-net adds more shallow features during decoding to yield a better DSC coefficient of $86.64 \%$. However, the characteristic information expressed is not obvious enough because the shallow features added in U-net is too simple, which results in obvious over-segmentation (as shown in Figure 5(b)). Deeplabv3+ achieves the highest detection accuracy, reaching $92.47 \%$, but its recall rate is only $86.00 \%$. This is because Deeplabv3+ utilizes multi-scale information fusion, which improves the accuracy of detection, but on the other hand, misses foregrounds to cause under-segmentation (Figure 5(d)). More specifically, the multi-scale information is redundant when the ischemic area is not obvious, which make the network less sensitive to fuzzy targets. This also results in that, although Deeplabv3+ shows a high detection precision rate of $92.47 \%$, the overall DSC coefficient and IOU coefficient are not as high as ours. As a variant of U-net structure, compared with U-net method, U-net++ achieves $89.46 \%$ and $85.68 \%$ in DSC coefficient and IOU coefficient, respectively. The reason can be attributed to the fact that long and short connections are used to extract different levels of features, and those features are integrated through superposition, which also overcomes the shortcomings of U-net's large fluctuations in the segmented DSC and IOU. However, U-net++ over-integrates different levels of features, which brings redundant features, and affects the sensitivity of feature expression.

Compared with the above segmentation method, the proposed method achieves the better balance between sensitivity and accuracy with the highest Recall, DSC and IOU (Table 1,2). The reason lies in the following three aspects: (1) We add a non-local attention module [13] to the encoder, which can employ the features extracted by the encoder to capture the characteristics of the dependence between long-distance features. Therefore, at the level of the extracted features, considering the bilateral differences, the false positive errors are reduced. As shown in Table 4, the recall rate improves from $90.70 \%$ to $95.59 \%$ when we have introduced non-local unit. (2) In the decoder, in order to improve feature sensitivity, we use the CIF module to fuse the characteristics of the channel dimensions and improve the expression of global information. The result show that DSC improved from $87.38 \%$ to $88.62 \%$ and the fluctuation has been reduced from $25.02 \%$ to $22.97 \%$ (Table 4). (3) We designed SIF module to extract the rich spatial features of the shallow encoder.
Compared with the simple integration of shallow information in Unet, this method reduces spatial feature redundancy and increases the precision rate from $80.23 \%$ to $88.25 \%$. It can also be found from Figure 5(f) that our method can basically segment the target area, but there is a certain degree of over-segmentation. Due to the improvement of the precision rate. the IOU improved from $84.32 \%$ and $86.75 \%$ when we used SIF unit.

The results of LHI detection in hyper-acute phase based on NCCT image show that the accuracy, precision, recall and ROC of the CAD method we proposed $91.67 \%, 90.95 \%, 91.67 \%$, and 0.89 , respectively. Each one is higher than the results obtained based on other existing segmentation methods. This shows that our precise segmentation network is conducive to improving the accuracy of the CAD program in detecting HLI and thus can reduce clinical false alarms.

The proposed method yields the best Recall, Precision, Accuracy, and AUC on LHI dataset, being respectively $91.67 \%, 90.95 \%, 91.67 \%$, and 0.89 , respectively (Table 5 ). This shows that the proposed segmentation network is conducive to improving the accuracy of the CAD program in detecting LHI.

This study is without limitations. First of all, the number of images is limited. In the future, we should collect more data and further enhance the model. Second, the proposed method balance between sensitivity and specificity, thus does not yield the highest precision (Table 1). In the future, we will work on improving precision.

In summary, in this study, we propose an algorithm for CAD of LHI at hyper-acute phase NCCT images. This method can automatically detect the ischemic area and recognize LHI patients. The proposed method utilizes a segmentation $\mathrm{CNN}$ algorithm based on "encoding-decoding". Compared with existing automatic segmentation methods, the proposed method can detect and segment the ischemia accurately. The proposed method provides a fast and effective means for research and clinical practice to quickly and accurately diagnose LHI in the hyper-acute phase, so as to provide method support for decision-making on thrombolysis or thrombectomy.

\section{REFERENCES}

[1] Powers W J, Rabinstein A A, Ackerson T, et al. 2018 guidelines for the early management of patients with acute ischemic stroke: a guideline for healthcare professionals from the American Heart Association/American Stroke Association[J]. Stroke, 2018, 49(3): e46-e99.

[2] Hacke W, Schwab S, Horn M, et al. 'Malignant'middle cerebral artery territory infarction: clinical course and prognostic signs[J]. Archives of neurology, 1996, 53(4): 309-315.

[3] Berrouschot J, Sterker M, Bettin S, et al. Mortality of space-occupying ('malignant') middle cerebral artery infarction under conservative intensive care[J]. Intensive care medicine, 1998, 24(6): 620-623. 
[4] Vahedi K, Hofmeijer J, Juettler E, et al. Early decompressive surgery in malignant infarction of the middle cerebral artery: a pooled analysis of three randomised controlled trials[J]. The Lancet Neurology, 2007, 6(3): 215-222.

[5] Zhao J, Su Y Y, Zhang Y, et al. Decompressive hemicraniectomy in malignant middle cerebral artery infarct: a randomized controlled trial enrolling patients up to 80 years old[J]. Neurocritical care, 2012, 17(2): 161-171.

[6] Torbey M T, Bösel J, Rhoney D H, et al. Evidence-based guidelines for the management of large hemispheric infarction[J]. Neurocritical care, 2015, 22(1): 146-164.

[7] Chen L, Bentley P, Rueckert D. Fully automatic acute ischemic lesion segmentation in DWI using convolutional neural networks[J]. NeuroImage: Clinical, 2017 , 15: 633-643

[8] Clèrigues A, Valverde S, Bernal J, et al. Acute ischemic stroke lesion core segmentation in CT perfusion images using fully convolutional neural networks[J] Computers in biology and medicine, 2019, 115: 103487.

[9] Tang F, Ng D K S, Chow D H K. An image feature approach for computer-aided detection of ischemic stroke[J]. Computers in biology and medicine, 2011, 41(7): 529-536.

[10] Boers AM, Marquering HA, Jochem JJ, et al. Automated cerebral infarct volume measurement in follow-up noncontrast $\mathrm{CT}$ scans of patients with acute ischemic stroke. Am J Neuroradiol 2013;34:1522-1527.

[11] Qiu W, Kuang H, Teleg E, et al. Machine learning for detecting early infarction in acute stroke with non-contrast-enhanced CT[J]. Radiology, 2020, 294(3): 638-644.

[12] He K, Zhang X, Ren S, et al. Delving deep into rectifiers: Surpassing human-level performance on imagenet classification[C]//Proceedings of The IEEE International Conference on Computer Vision. 2015: 1026-1034.

[13] Wang X, Girshick R, Gupta A, et al. Non-local neural networks[C]//Proceedings of The IEEE Conference on Computer Vision and Pattern Recognition. 2018: 7794-7803.
[14] Ioffe S, Szegedy C. Batch normalization: Accelerating deep network training by reducing internal covariate shift[J]. arXiv preprint arXiv:1502.03167, 2015

[15] Kuang H, Menon B K, Qiu W. Semi-automated infarct segmentation from followup noncontrast CT scans in patients with acute ischemic stroke[J]. Medical Physics, 2019, 46(9): 4037-4045.

[16] Wang X, Girshick R, Gupta A, et al. Non-local neural networks[C]//Proceedings of The IEEE Conference on Computer Vision and Pattern Recognition. 2018: 7794-7803.

[17] $\mathrm{Hu}$ J, Shen L, Sun G. Squeeze-and-excitation networks[C]//Proceedings of The IEEE Conference on Computer Vision and Pattern Recognition. 2018: 7132-7141.

[18] Zeiler M D, Fergus R. Visualizing and understanding convolutional networks[C]//European Conference on Computer Vision. Springer, Cham, 2014: 818-833.

[19] Abraham N, Khan N M. A novel focal tversky loss function with improved attention u-net for lesion segmentation[C]//2019 IEEE 16th International Symposium on Biomedical Imaging (ISBI 2019). IEEE, 2019: 683-687.

[20] Kingma D P, Ba J. Adam: A method for stochastic optimization[J]. arXiv preprint arXiv:1412.6980, 2014.

[21] Badrinarayanan V, Kendall A, Cipolla R. Segnet: A deep convolutional encoderdecoder architecture for image segmentation[J]. IEEE Transactions on Pattern Analysis and Machine Intelligence, 2017, 39(12): 2481-2495.

[22] Chen L C, Zhu Y, Papandreou G, et al. Encoder-decoder with atrous separable convolution for semantic image segmentation[C]//Proceedings of The European Conference on Computer Vision (ECCV). 2018: 801-818.

[23] Zhou Z, Siddiquee M M R, Tajbakhsh N, et al. Unet++: A nested u-net architecture for medical image segmentation[M]//Deep Learning in Medical Image Analysis and Multimodal Learning for Clinical Decision Support. Springer, Cham, 2018: 3-11. 